\title{
Morphological Interrelationship Between Astrocytes and Nerve Endings in the Rat Spinal Trigeminal Nucleus Caudalis
}

\author{
By \\ Zhang YIHONG ${ }^{1}$, Yoshitaka TAMADA ${ }^{2}$, Keisuke AKAI ${ }^{2}$ and Fumihiko SUWA ${ }^{2}$ \\ ${ }^{1}$ The Health Administration of PLA, FuXing Road N0.22 A3, 100842, Beijing, P.R. China \\ ${ }^{2}$ Department of Anatomy, Osaka Dental University, 8-1 Kuzuhahanazono-cho, Hirakata-shi, \\ Osaka 573-1121, Japan
}

- Received for Publication, September 7, 2006-

Key Words: Spinal trigeminal nucleus caudalis, Astrocytes, Electron microscopic immunohistochemistry, Nerve endings, Rat

\begin{abstract}
Summary: It has been reported that the spinal trigeminal nucleus caudalis (Sp5C), which receives nociceptive information from the oro-facial regions, has four laminae. To clarify the role of glial cells in the transmission of the nociceptive information, the present study was conducted to examine the detailed distribution of astrocytes in each lamina and also to investigate a morphological interrelationship between the astrocytes and nerve endings in the rat Sp5C. After the preparation of the serial cryostat sections, immunohistochemistry for glial fibrillary acidic protein (GFAP) was employed to identify the astrocytes, and immunohistochemistry for substance $P$ (SP), calcitonin gene-related peptide (CGRP), was used for the nerve endings. We also employed double-labeling immunofluorescence and electron microscopic immunohistochemistry for the GFAP/SP or GFAP/CGRP. GFAP-positive reactions were observed in all laminae of the Sp5C, and SP- or CGRP-positive nerve endings were observed in the lamina I and II. Additionally, we clarified the presence of GFAP/SP- or GFAP/CGRP-positive reactions by the double-labeling immunofluorescence and demonstrated the morphological interrelationship between the astrocytes and nerve endings by the double-labeling electron microscopic immunohistochemistry. These findings suggest that astrocytes might play some roles in the transmission of nociceptive information from the oro-facial region.
\end{abstract}

Spinal trigeminal nucleus caudalis (Sp5C) plays important roles in the transmission of the nociceptive information from the oro-facial regions ${ }^{1-4)}$. The Sp5C is divided into four laminae (I-IV) from the outside layer to the layer on the inside, sequentially ${ }^{5)}$. Additionally, lamina I and II of the Sp5C receive the neuronal inputs of the primary afferent nerve endings, which contain neurotransmitters such as calcitonin gene-related peptide (CGRP) and substance P (SP) ${ }^{6,7)}$.

Recently, it has been reported that glial cells may be involved in the transmission of the nociceptive information ${ }^{8)}$. In the Sp5C, Hajos et al. demonstrated the presence of astrocytes using the immunohistochemistry (IHC) for glial fibrillary acidic protein (GFAP) ${ }^{9)}$ that is a specific marker for astrocytes ${ }^{10)}$. However, the detailed morphological investigations between the astrocytes and the nerve endings have not yet clarified.
In the present study, IHC for GFAP was employed to investigate the detailed distribution of astrocytes in each lamina of the Sp5C. Furthermore, double-labeling immunofluorescence (IF) and electron microscopic immunohistochemistry (EMIHC) for GFAP/SP or GFAP/CGRP was also employed to investigate a morphological interrelationship between the astrocytes and the nerve endings.

\section{Materials and Methods}

A total of thirty-two male Wistar rats (8 weeks after birth; purchased from Shimizu laboratory Supplies, Kyoto Japan) were used in the present study. These animals were housed in a temperaturecontrolled room $\left(23-25^{\circ} \mathrm{C}\right)$. They were maintained in individual cages under a light/dark cycle (lights on at 0800, lights off at 2000) and were given free 
access to food and water. The present study was conducted according to the Guidelines for Animal Research at Osaka Dental University (ODU) and the National Institute of Health (NIH) Guideline for the Care and Use of Laboratory Animals, and the study was approved by the Animal Research Committee for ODU (approval number; 05-06002).

\section{A. Light Microscopic Investigation}

All of the animals were sacrificed by an overdose injection of pentobarbital $(50 \mathrm{mg} / \mathrm{kg}$, i.p.; Nembutal $^{\circledR}$, Dainippon Pharmaceutical Co. Ltd) and immediately were perfused via the left cardiac ventricle with a $0.1 \mathrm{M}$ phosphate-buffered saline (PBS) and a fixative containing $4 \%$ paraformaldehyde, which was adjusted to $\mathrm{pH} 7.4$ with $0.1 \mathrm{M}$ phosphate buffer (PB). After the removal of the brain, tissue blocks including the Sp5C were post-fixed in the same fixative for $24 \mathrm{~h}$ at $4{ }^{\circ} \mathrm{C}$, and then immersed in $20 \%$ sucrose in $0.1 \mathrm{M} \mathrm{PB}$ for $24 \mathrm{~h}$ at $4{ }^{\circ} \mathrm{C}$.

Serial, free-floating frontal sections $(30 \mu \mathrm{m}$ in thickness) were prepared with a cryostat (MICROM HM500-OM ${ }^{\circledR}$, ZEISS) and the following histological procedures were performed.

\section{Nissl staining}

To investigate the cellular location in the Sp5C, sections obtained from four animals were used for Nissl staining in a $0.1 \%$ cresyl violet (Cresyl violet ${ }^{\circ}$, Chroma) solution.

\section{Immunohistochemistry}

A total of twelve animals were used for the IHC, and divided into three groups of four animals. Using an enzyme-labeled polymer method (EnVision + system $^{\circledR}$, DAKO), IHC was employed on the sections obtained from the animals in these three groups to analyze the detailed distribution of the GFAP, SP and CGRP, respectively. The sections were incubated in a mouse monoclonal anti-GFAP (dilution 1:1000, SIGMA), a rabbit polyclonal antiSP (dilution 1:3000, CHEMICON) or a rabbit polyclonal anti-CGRP (dilution 1:3000, Amersham) serum diluted in a $0.1 \mathrm{M}$ PBS containing $0.1 \%$ Triton $\mathrm{X}-100$ overnight at $4{ }^{\circ} \mathrm{C}$. After rinsing, they were incubated with a secondary antibody (Labeled polymer, $\left.\mathrm{HRP}^{\circledR}, \mathrm{DAKO}\right)$ for $1 \mathrm{~h}$ at room temperature. Next, they were exposed to a $50 \mathrm{mM}$ Tris$\mathrm{HCl}$ buffer containing $0.01 \%$ 3-3'-diaminobenzidine $4 \mathrm{HCl}(\mathrm{DAB})$ and $0.005 \% \mathrm{H}_{2} \mathrm{O}_{2}$.

\section{Double-labeling immunofluorescence}

To investigate a morphological interrelationship between the astrocytes and the nerve endings, a total of eight animals were used for double-labeling
IF, and divided into two GFAP/SP- and GFAP/ CGRP-groups of four animals.

The sections were incubated with (1) GFAP/SP (dilution GFAP 1:1000; SP, 1:3000) or (2) GFAP/ CGRP (dilution GFAP 1:1000; CGRP, 1:3000) serum diluted in a $0.1 \mathrm{M}$ PBS containing $0.1 \%$ Triton $\mathrm{X}-100$ overnight at $4^{\circ} \mathrm{C}$. And they were incubated with a $0.1 \mathrm{M}$ PBS containing fluoresceinisothiocyanate (FITC)-labeled anti-mouse $\mathrm{IgG}$ [FLORESCEIN ANTI-MOUSE $\operatorname{IgG}(\mathrm{H}+\mathrm{L})^{\circledR}$, dilution 1:1000, VECTOR] and tetrametylrhodamineisothyocyanate (TRITC)-labeled anti-rabbit IgG (Dky X Rbt IgG RHODAMINE ${ }^{\circledR}$, dilution 1:1000, CHEMICON) for $4 \mathrm{~h}$ at room temperature. After the immuno-reaction, the Nissl staining was performed in the same section to determine the expression lamina of the GFAP/CGRP- or GFAP/SPpositive reactions in the $\mathrm{Sp} 5 \mathrm{C}$.

The sections that underwent the Nissl staining and the IHC were examined with a light microscope (MICROPHOT-FXA ${ }^{\circledR}$, Nikon) and those with the double-labeling IF with a fluorescence microscope (BX50 ${ }^{\circledR}$, OLYMPUS).

\section{B. Electron Microscopic Investigation}

For the electron microscopic investigation, a total of eight animals were used for double-labeling EMIHC, and divided into two GFAP/SP- and GFAP/CGRP-groups of four animals. In the present study, GFAP-positive reactions were visualized by $\mathrm{DAB}$ with silver-gold intensification (DABSGI), and SP- or CGRP-positive reactions were detected by $\mathrm{DAB}$ alone.

After the perfusion with a fixative containing $4 \%$ paraformaldehyde and $0.35 \%$ glutaraldehyde in 0.1 M PB (pH7.4), the tissue blocks were post-fixed in a fixative containing $4 \%$ paraformaldehyde in $0.1 \mathrm{M} \mathrm{PB}(\mathrm{pH} 7.4)$ for $24 \mathrm{~h}$ at $4^{\circ} \mathrm{C}$. Serial frontal sections $(40 \mu \mathrm{m}$ in thickness) were cut with a microslicer (VIBRATOME Series 3000 SECTIONING SYSTEM ${ }^{\circledR}$, TECHNICAL PRODUCTS INTERNATIONAL INC.). The sections were first incubated with the anti-GFAP serum as previously mentioned, and then processed for the DAB-SGI according to the method reported by Liposits et $a l .{ }^{11)}$ In the next step, the sections were further incubated with the anti-SP or the anti-CGRP serum for $72 \mathrm{~h}$, and treated with a avidin-biotin complex (ABC) method.

After the immuno-reaction, the sections were fixed in chilled $1 \% \mathrm{OsO}_{4}$ solution for $1 \mathrm{~h}$ at $4{ }^{\circ} \mathrm{C}$, dehydrated through a graded series of acetone and embedded in Epon812 mixture (LUVEAK ${ }^{\circledR}$, nacalai tesque). Ultrathin sections were cut with a ultramicrotome (LEICA ULTRA UCT ${ }^{\circledR}$, Nissei Sangyo co Ltd.), stained with uranyl acetate, and 
examined with a transmission electron microscope (H-7100 ${ }^{\circledR}$, HITACHI).

The specificity of the anti-GFAP, the anti-SP and the anti-CGRP serum was confirmed by the preabsorption with synthetic peptide respectively. We also confirmed that cross-reaction in the doublelabeling IHC did not occurred using negative control serum (Universal Negative Control-Mouse, Rabbit ${ }^{\circledR}$, DAKO).

\section{Results}

\section{Nissl staining}

The location of the Sp5C could be identified by the Nissl staining (Fig. 1). In the higher magnification view, four laminae were observed in the $\mathrm{Sp} 5 \mathrm{C}$ (Fig. 3B, 3D). However, the borderline between lamina III and lamina IV was not obvious (Fig. 3B, 3D).

\section{Immunohistochemistry}

\section{(1) GFAP}

GFAP-positive reactions were observed throughout all of the Sp5C lamina, and especially in lamina I and II (Fig. 2A, 2D).

\section{(2) $S P$ and CGRP}

SP- or CGRP-positive reactions were demonstrated only in lamina I and II of the Sp5C. SP- or

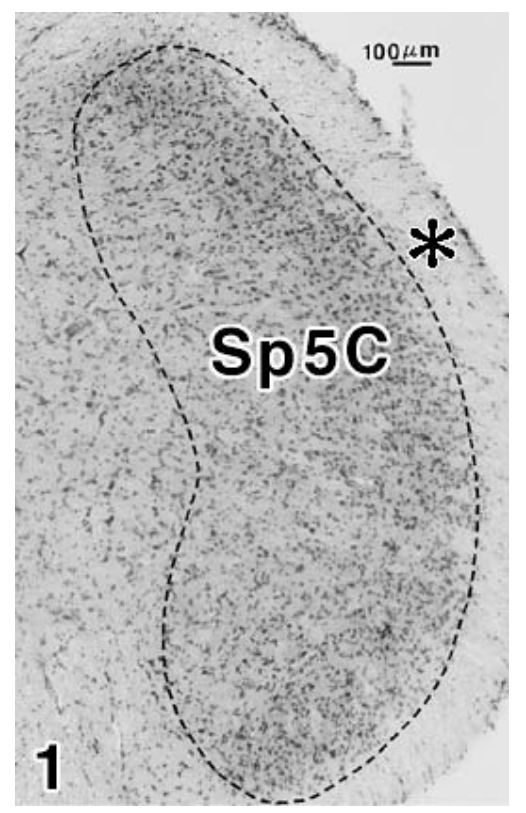

Fig. 1. Nissl staining section of the Sp5C. The location of the $\mathrm{Sp} 5 \mathrm{C}$ was identified in the Nissl-stained section. *: spinal trigeminal tract, broken line: Sp5C
CGRP-positive nerve endings were identified as small dots, and a large number of CGRP-positive nerve endings were observed, in comparison with the SP-positive nerve endings (Fig. 2B, 2C, 2E, 2F).

\section{Double-labeling immunofluorescence}

FITC-labeled astrocytic cell bodies and processes (green-colored) were showed a cobweb-like shape with processes extending in all directions (Fig. 3A, 3C). According to the simultaneous visualization of the IF and the Nissl staining (Fig. 3B, $3 \mathrm{D})$ in the same section, we observed GFAPpositive astrocytes (FITC-labeled; green-colored) and SP- or CGRP-positive nerve endings (TRITClabeled; red-colored) in lamina I and II (Fig. 3A, $3 \mathrm{C})$.

\section{Double-labeling electron microscopic immunohistochemistry}

In the lamina I and II of the Sp5C, many GFAPpositive processes with DAB-SGI were observed. And theses astrocytic processes made the direct contacts with the SP- or CGRP-positive nerve endings with DAB alone (Fig. 4A, 4B).

\section{Discussion}

As shown in Fig. 1, we identified four laminae of the Sp5C according to the existence of the large neurons ${ }^{5}$. However, the border line between the lamina III and IV was not obvious ${ }^{5)}$. Based on these laminar structures, the distribution of the astrocytes in the $\mathrm{Sp} 5 \mathrm{C}$ and the morphological interrelationship between the astrocytes and nerve endings were discussed.

\section{Distribution of the astrocytes in the Sp5C}

Our IHC results clarified that many astrocytes were located in lamine I and II, which received nociceptive information from oro-facial regions. Additionally, other studies have shown the presence of SP- or CGRP-positive nerve endings in lamina I and II of the Sp5C ${ }^{12,13)}$, and our study obtained the same results. Therefore, it was apparent that astrocytes were distributed in the region, which contained many SP- or CGRP-positive nerve endings.

\section{Morphological interrelationship between astrocytes and nerve endings}

We have previously demonstrated the presence of astrocytic contacts with other nerve endings in the other regions of the brain ${ }^{14,15)}$. In the present study, using the double-labeling IF and EMIHC, dense SP- or CGRP-positive reactions were observed around the GFAP-positive reactions, and we 


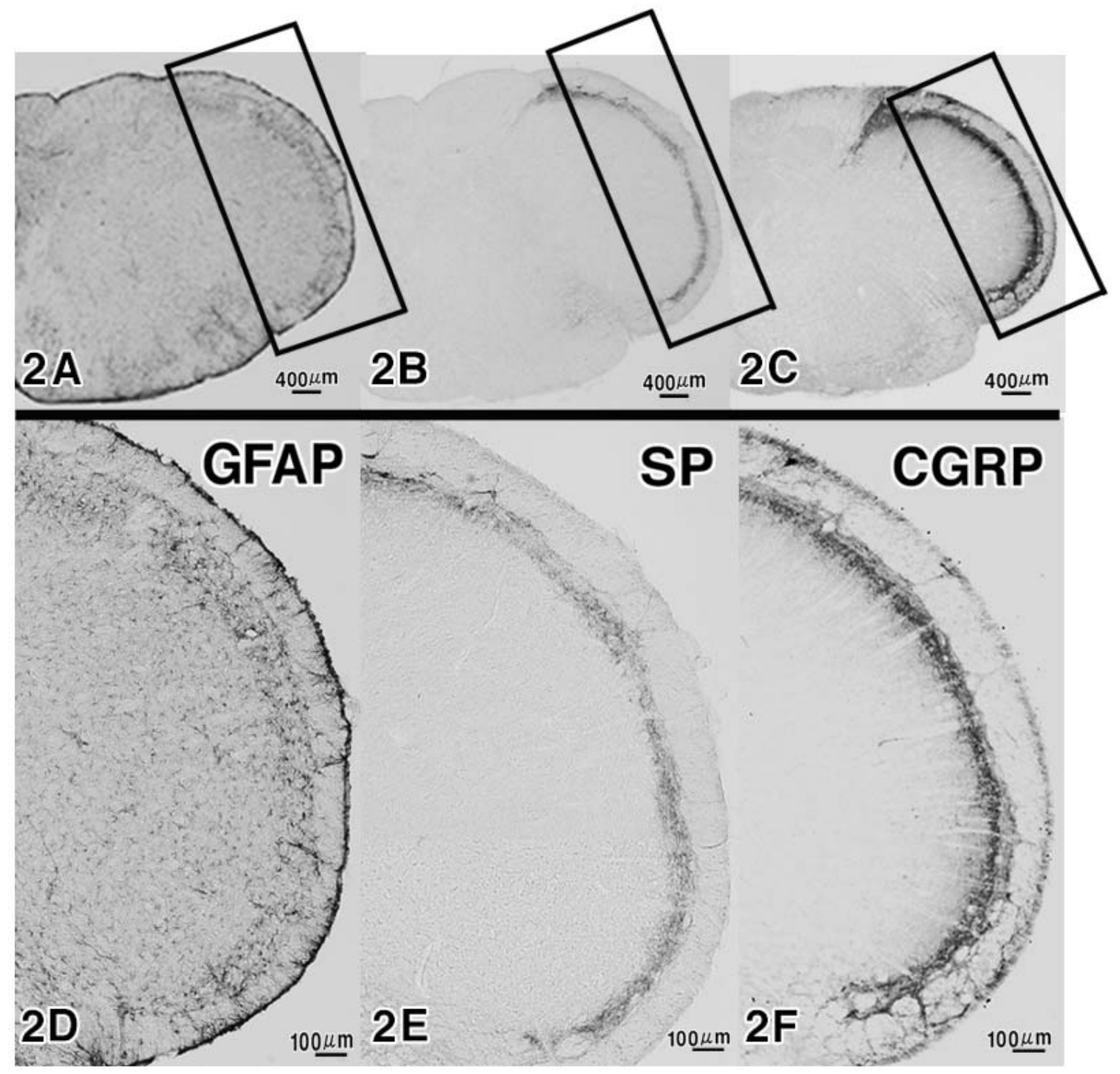

Fig. 2. Immunohistochemistry for GFAP, SP and CGRP. Positive reactions for GFAP (2A), SP (2B) or CGRP (2C) were observed in the Sp5C. Fig. 2D, 2E and 2F indicate a higher magnification view of square regions in Fig. 2A, 2B and C, respectively. GFAP-positive reactions were observed in all laminae of the Sp5C, and SP- or CGRP-positive nerve endings were observed in the lamina I and II.

also clarified the morphological contact between astrocytic processes and SP- or CGRP-positive nerve endings in the lamina I and II of the Sp5C.

It has also been reported that astrocytes, which support and nourish the neurons, also regulate extra-cellular ion concentrations and release some substances into the extra-cellular space in response to various kinds of neurotransmission ${ }^{16-20}$. Additionally, it has been demonstrated that some physiological stimuli such as aging and the light-dark conditions cause morphological and functional changes to astrocytes ${ }^{21,22)}$.
In addition, the following findings have been reported: 1. Astrocytes are activated within the thoracic spinal cord following the intramyocardial injection of formalin ${ }^{8)}$, 2. In spinal astrocytes, pain stimulation to the nerve endings induces changes in some kinds of substances related to the cellular signal transduction ${ }^{23)}, 3$. In the process of the nociceptive information transmission, SP in the nerve endings are related to the synthesis of the prostaglandins in the astrocytes ${ }^{24)}$, and 4 . SP- and CGRPreceptors exist in the astrocytes ${ }^{25,26)}$. Therefore, our morphological results suggested that astrocytes 


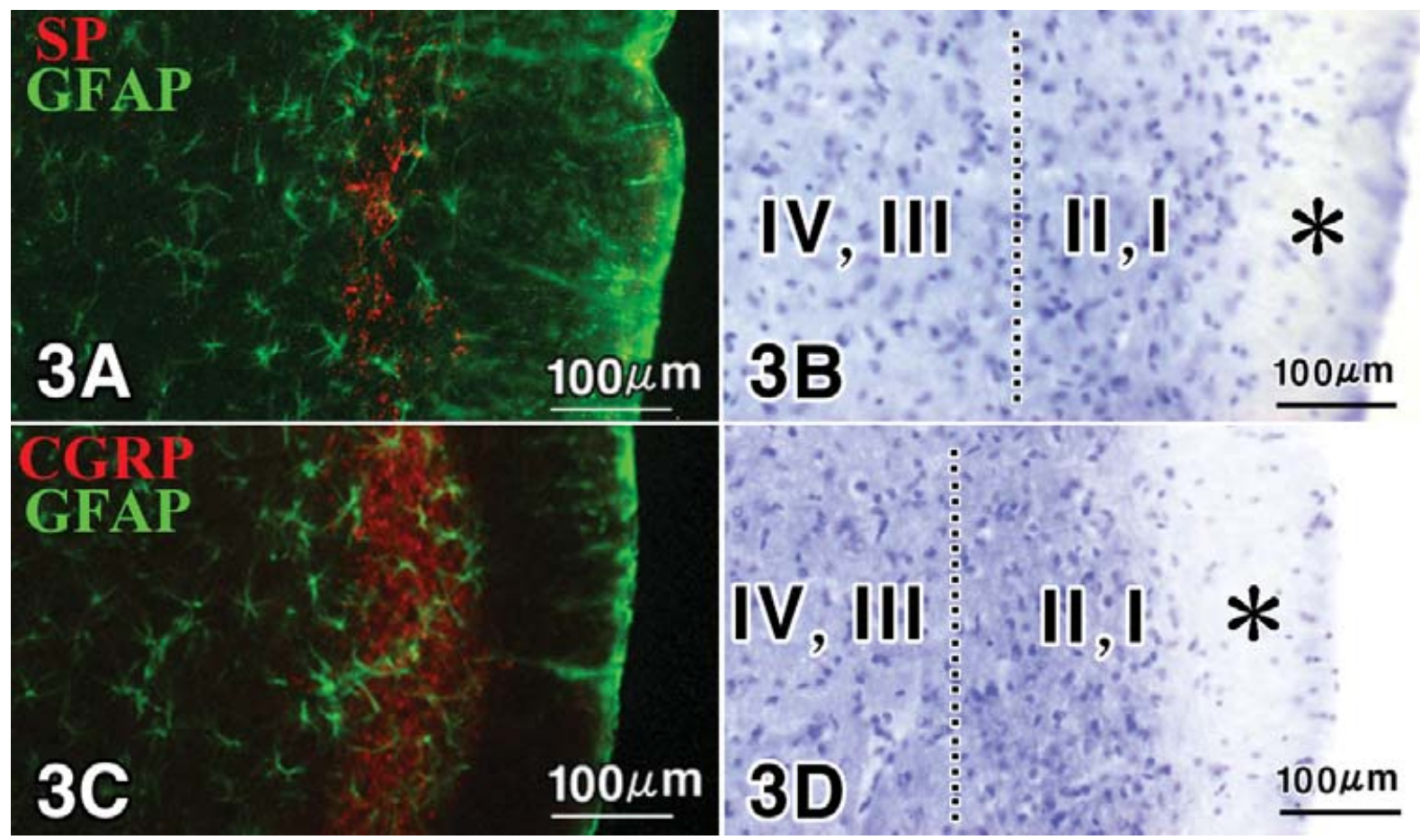

Fig. 3. Double-labeling immunofluorescence. Four laminae were identified in the Sp5C (3B, 3D). GFAP/SP (3A)-, or GFAP/CGRP (3C)-positive reactions were observed in lamina I and II in the Nissl staining sections of the Sp5C (3B, 3D).

I, II, III, IV: lamina I-IV (from the outside lamina I to the inside lamina IV, sequentially), *: spinal trigeminal tract
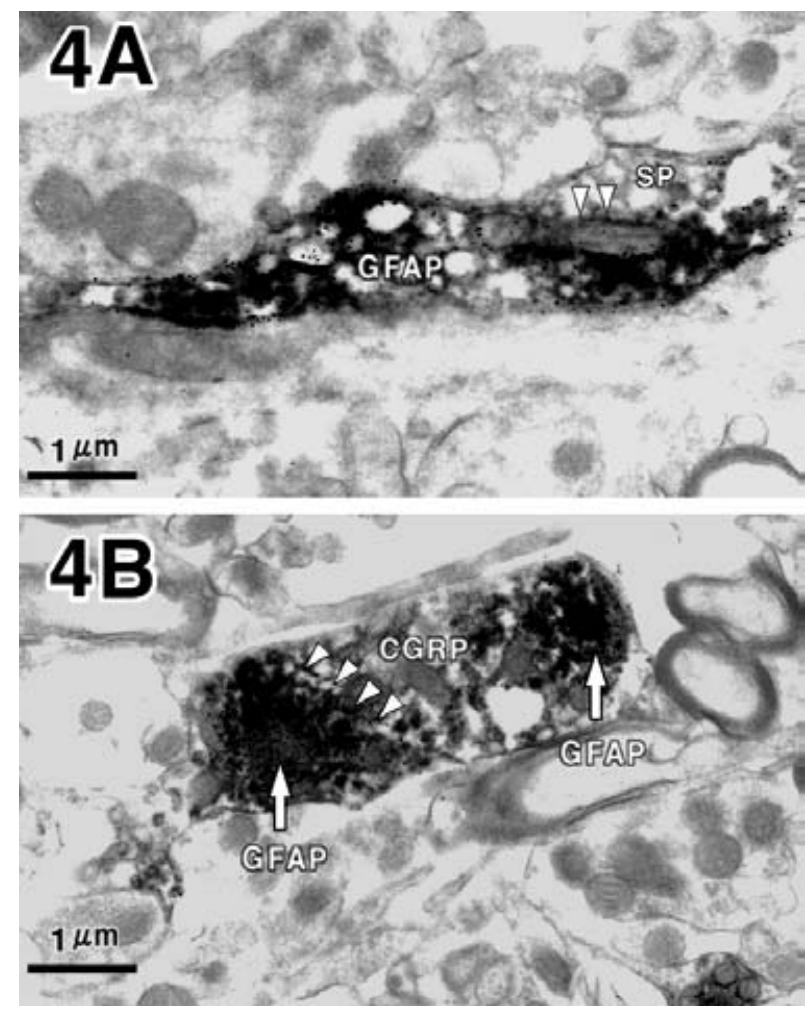

in the $\mathrm{Sp} 5 \mathrm{C}$ might receive the nociceptive information from the primary afferent nerve endings, and also that they might play some important roles in the transmission of the nociceptive information from the oro-facial regions.

\section{Acknowledgments}

This study was performed using the Laboratory Animal Facilities and the Morphological Research Facilities of the Institute of Dental Research, Osaka Dental University.

Fig. 4. Double-labeling electron microscopic immunohistochemistry. In the lamina I and II, GFAP-positive astrocytic processes with DAB-SGI were observed to make direct contacts with SP(4A)- or CGRP(4B)-positive nerve endings with $\mathrm{DAB}$ alone.

DAB: 3-3'-diaminobenzidine 4HCl, DAB-SGI: DAB with silver-gold intensification 


\section{References}

1) Bernard JF and Besson JM. The spino(trigemino)pontoamygdaloid pathway: electrophysiological evidence for an involvement in pain processes. J Neurophysiol 1990; 63:473-490.

2) Bester H, Menendez L, Besson JM and Bernard JF. Spino (trigemino) parabrachiohypothalamic pathway: electrophysiological evidence for an involvement in pain processes. J Neurophysiol 1995; 73:568-585.

3) DeSalles AA, Katayama Y, Becker DP and Hayes RL. Pain suppression induced by electrical stimulation of the pontine parabrachial region. Experimental study in cats. J Neurosurg 1985; 62:397-407.

4) Dubner R and Bennett GJ. Spinal and trigeminal mechanisms of nociception. Annu Rev Neurosci 1983; 6:381-418.

5) Waite P and Tracey D. Trigeminal Sensory System. In: G Paxinos, The Rat Nervous System, New York, Academic Press, 1995; 705-724.

6) Otsuka $\mathrm{M}$ and Yanagisawa M. Pain and neurotransmitters. Cell Mol Neurobiol 1990; 10:293-302.

7) Todd AJ and Spike RC. The localization of classical transmitters and neuropeptides within neurons in laminae I-III of the mammalian spinal dorsal horn. Prog Neurobiol 1993; 41:609-645.

8) Ping Dai R, Ping He B, Thameem Dheen S and Tay SS. Acute cardiac injury induces glial cell response and activates extracellular signaling-regulated kinase- 1 and -2 in the spinal cord of Wistar rats. Neurosci Lett 2004; 366:3438.

9) Eng LF, Vanderhaeghen JJ, Eignami A and Gerstil B. An acidic protein isolated from fibrous astrocytes. Brain Res 1971; 28:351-354.

10) Hajos F and Kalman M. Distribution of glial fibrillary acidic protein (GFAP)-immunoreactive astrocytes in the rat brain: II. Mesencephalon, rhombencephalon and spinal cord. Exp Brain Res 1989; 78:164-173.

11) Liposits Z, Sherman D, Phelix C and Paull WK. A combined light and electron microscopic immunocytochemical method for the simultaneous localization of multiple tissue antigens. Tyrosine hydroxylase immunoreactive innervation of corticotropin releasing factor synthesizing neurons in the paraventricular nucleus of the rat. Histochemistry 1986; 85:95-106.

12) Cuello AC and Kanazawa I. The distribution of substance P immunoreactive fibers in the rat central nervous system. J Comp Neurol 1978; 178:129-156.

13) Wiesenfeld-Hallin Z, Hokfelt T, Lundberg JM, Forssmann WG, Reinecke M, Tschopp FA and Fischer JA. Immunoreactive calcitonin gene-related peptide and substance $\mathrm{P}$ coexist in sensory neurons to the spinal cord and interact in spinal behavioral responses of the rat. Neurosci Lett 1984; 52:199-204

14) Tamada Y, Hayshi S, Munekawa K, Tanaka M, Ikeda T, Inoue $\mathrm{K}$ and Ibata $\mathrm{Y}$. Morphological interaction between glial fibrillary acidic protein (GFAP)-like immunoreactive elemnts and lutenizing hornone releasing hormone (LHRH)-like immunoreactive nerve endings in the median eminence of the rat - Double labeling immunoelectron microscopic study -. Acta Histochem Cytochem 1997; 30:517-523.

15) Tamada Y, Tanaka M, Munekawa K, Hayashi S, Okamura H, Kubo T, Hisa Y and Ibata Y. Neuron-glia interaction in the suprachiasmatic nucleus: a double labeling light and electron microscopic immunocytochemical study in the rat. Brain Res Bull 1998; 45:281-287.

16) Nedergaard M. Direct signaling from astrocytes to neurons in cultures of mammalian brain cells. Science 1994; 263:1768-1771.

17) Teichberg VI. Glial glutamate receptors: likely actors in brain signaling. Faseb J 1991; 5:3086-3091.

18) Sontheimer H, Black JA and Waxman SG. Voltage-gated $\mathrm{Na}+$ channels in glia: properties and possible functions. Trends Neurosci 1996; 19:325-331.

19) Zhou M, Tanaka O, Suzuki M, Sekiguchi M, Takata K, Kawahara $\mathrm{K}$ and Abe $\mathrm{H}$. Localization of pore-forming subunit of the ATP-sensitive $\mathrm{K}(+)$-channel, Kir6.2, in rat brain neurons and glial cells. Brain Res Mol Brain Res 2002; 101:23-32.

20) $\mathrm{Ng} \mathrm{CH}$, Wang XS and Ong WY. A light and electron microscopic study of the GABA transporter GAT-3 in the monkey basal ganglia and brainstem. J Neurocytol 2000; 29:595-603.

21) Ike H, Tamada $Y$, Uemura $M$, Ishihara $A$, Suwa $F$ and Ibata Y. Age-related changes in astrocytes and microvasculature in the median eminence of the rat. Acta Histochem Cytochem 2004; 37:129-138.

22) Munekawa K, Tamada $Y$, Iijima N, Hayashi S, Ishihara A, Inoue K, Tanaka M and Ibata Y. Development of astroglial elements in the suprachiasmatic nucleus of the rat: with special reference to the involvement of the optic nerve. Exp Neurol 2000; 166:44-51.

23) $\mathrm{Ma} \mathrm{W}$ and Quirion R. Partial sciatic nerve ligation induces increase in the phosphorylation of extracellular signal-regulated kinase (ERK) and c-Jun N-terminal kinase (JNK) in astrocytes in the lumbar spinal dorsal horn and the gracile nucleus. Pain 2002; 99:175-184.

24) Marriott D, Wilkin GP, Coote PR and Wood JN. Eicosanoid synthesis by spinal cord astrocytes is evoked by substance $\mathrm{P}$; possible implications for nociception and pain. Adv Prostaglandin Thromboxane Leukot Res 1991; 21B:739-741.

25) Guo CJ, Douglas SD, Gao Z, Wolf BA, Grinspan J, Lai JP, Riedel $\mathrm{E}$ and Ho WZ. Interleukin-1beta upregulates functional expression of neurokinin-1 receptor (NK-1R) via NF-kappaB in astrocytes. Glia 2004; 48:259-266.

26) Haas CA, Reddington $M$ and Kreutzberg GW. Calcitonin Gene-related Peptide Stimulates the Induction of c-fos Gene Expression in Rat Astrocyte Cultures. Eur J Neurosci 1991; 3:708-712. 